\title{
Flora e estrutura do estrato lenhoso numa comunidade de Cerrado em Santo Antônio do Leverger, MT, Brasil
}

\author{
HÉLIDA B. NOGUEIRA BORGES ${ }^{1,3}$ e GEORGE J. SHEPHERD ${ }^{2}$
}

(recebido: 4 de setembro de 2004; aceito: 5 de novembro de 2004)

\begin{abstract}
Flora and structure of woody layer inside of a "cerrado" community in Santo Antônio do Leverger, MT, Brazil). An analysis was made of the floristic composition and structure of the shrub-arboreous layer of the "cerrado" community in Mato Grosso. A total of 131 species, 92 genera and 45 families was recorded for this area. The families richest in species were: Leguminosae, Myrtaceae, Rubiaceae, Annonaceae and Malpighiaceae, with 43\% (56/131) of the species and 38\% (35/92) of the genera. Date analysis about floristic similarity, by Jaccard Index (IJ) and Sørensen Index (IS), with 20 other "cerrado" (sensu lato) sites showed that the area studied has the most species in common with Mato Grosso and Pantanal vegetation. The area studied had high species diversity $\left(\mathrm{H}^{\prime}=3,75\right.$ nats.individual $\left.{ }^{-1}\right)$. The structural survey recorded total density of 1,653 individuals. ha $^{-1}$. The most important species was Annona dioica A.St.-Hil. and the most important family was Annonaceae. The importance of the most species was litle, considering the values of density, frequency and dominance. These results, associated to the fact that the community suffer constant environmental disturbance, justify the studies and step taken of conservation in a short period of time for the studied area.
\end{abstract}

Key words - cerrado, conservation, floristic composition, phytosociology, woody species

RESUMO - (Flora e estrutura do estrato lenhoso numa comunidade de cerrado em Santo Antônio do Leverger, MT, Brasil). Numa comunidade de cerrado foram desenvolvidos estudos quanto à composição da flora e características estruturais do estrato arbustivo-arbóreo. Um total de 131 espécies, 92 gêneros e 45 famílias foram registrados na área. As famílias com maior riqueza de espécies foram: Leguminosae, Myrtaceae, Rubiaceae, Annonaceae e Malpighiaceae, com 43\% (56/131) das espécies e 38\% (35/92) dos gêneros. Análise de dados sobre a similaridade florística, pelos índices de Jaccard (IJ) e Sørensen (IS), com 20 outros locais de cerrado (sensu lato) revelou que a área estudada tem mais espécies em comum com a vegetação de Mato Grosso e do Pantanal. A área estudada apresentou alta diversidade de espécies $\left(\mathrm{H}^{\prime}=3,75\right.$ nats.individuo $\left.{ }^{-1}\right)$. Na pesquisa

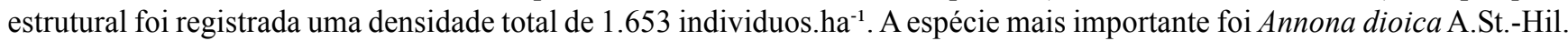
e a família foi Annonaceae. A importância da maioria das espécies foi pequena, considerando os valores de densidade, freqüência e dominância. Esses resultados, associados ao fato que a comunidade sofre perturbações ambientais constantes, justificam estudos e medidas de conservação em curto prazo para a área estudada.

Palavras-chave - cerrado, conservação, composição florística, espécies lenhosas, fitossociologia

\section{Introdução}

O cerrado, a segunda maior formação vegetal do Brasil, apresenta grande diversidade fisionômica e florística em seus domínios. As variações fisionômicas do cerrado produzem um gradiente, em densidade e altura, definido por formações campestres a florestais (Coutinho 1978). O cerrado reúne a mais importante flora savânica dos neotrópicos (Sarmiento 1983). Estimativas apontam entre 1.000 a 2.000 espécies arbustivo-arbóreas e 2.000 a 5.250 herbáceosubarbustivas compondo a flora (Castro et al. 1999).

\footnotetext{
1. Universidade Federal de Mato Grosso, Herbário Central, Av. Fernando Corrêa da Costa s/n, 78060-900 Cuiabá, MT, Brasil.

2. Universidade Estadual de Campinas, Instituto de Biologia, Departamento de Botânica, Caixa Postal 6109, 13081-100 Campinas, SP, Brasil; george@unicamp.br

3.Autor para correspondência: hbruno@terra.com.br
}

Análise florística da vegetação do cerrado revela heterogeneidade entre áreas, caracterizada por associação particular de espécies (Ratter et al. 1996, 2003, Castro et al. 1999). As diferenças florísticas independem das distâncias que separam as áreas (Felfili \& Silva Junior 1993). As áreas diferem possivelmente como conseqüência das diferenças fisionômicas, das variações no solo, da disponibilidade de água no lençol freático, da influência do fogo, da substituição de estágios serais para cerradão ou floresta, dos fatores estocásticos ou relacionados à distribuição das espécies (Ribeiro et al. 1985, Furley \& Ratter 1988, Ratter \& Dargie 1992), da precipitação total e do comprimento da estação seca (Toledo Filho et al. 1989, Ratter et al. 1996).

A vegetação de cerrado ocupa o Planalto Central brasileiro e áreas periféricas na região norte, nordeste e sudeste e tem como ecossistemas adjacentes as florestas, os campos rupestres, os campos úmidos, os brejos permanentes e o Pantanal (Eiten 1994). No 
Pantanal, que se estende pelos Estados de Mato Grosso e Mato Grosso do Sul, a flora do cerrado ocupa 70\% da superfície (Adámoli 1981).

O cerrado constitui, assim, um grande mosaico, onde as peças são definidas pelas diferenças fisionômicas, florísticas e tipos de vegetação associados. Essas características, inerentes à vegetação, devem ser consideradas ao se definir programas de conservação para a região (Felfili \& Silva Junior 1993, Ratter et al. 1997). O conhecimento do cerrado implica no estudo das frações do mosaico, definindo suas características e como se relacionam. O primeiro passo nesse sentido está na determinação da composição de espécies e da forma como as mesmas estruturam a comunidade no espaço.

Estudos florísticos e fitossociológicos foram desenvolvidos em vegetação de cerrado em diferentes regiões do país, sendo que para Mato Grosso as pesquisas apontam significativa riqueza florística (Ratter et al. 2003). Entretanto, como em outras áreas de cerrado, essa riqueza está sendo perdida rapidamente pela substituição da vegetação natural por monoculturas agrícolas, pastagens, desmatamentos, queimadas e urbanização (Ferreira \& Monteiro 1990, Azevedo et al. 1995, Ratter et al. 1997, Furley 1999). Imagens de satélites foram usadas para estimar a integridade da vegetação nativa do cerrado (sensu lato) e os resultados sugerem que somente um terço das áreas se encontra pouco antropizado (BRASIL 1999). Nesse estudo foi evidenciado em Mato Grosso, junto com Goiás, São Paulo e Mato Grosso do Sul, apresentam de 50\% a 92\% do cerrado fortemente antropizado.

O presente trabalho tem como objetivo conhecer a flora lenhosa e características estruturais do estrato arbustivo-arbóreo numa área de cerrado em Mato Grosso. Os resultados deverão contribuir para ampliar o conhecimento sobre a vegetação do cerrado, quanto à sua diversidade florística e estrutural e, ainda, influenciar na formulação de programas de manejo e na definição de medidas de preservação para a vegetação na região.

\section{Material e métodos}

Descrição da Área - Os estudos foram desenvolvidos em 4,9 ha de cerrado (sensu stricto, Coutinho 1978) que representa o maior espaço com vegetação natural nos 210,4 ha da Fazenda Experimental da Universidade Federal de Mato Grosso (UFMT). AFazenda Experimental ( $\left(15^{\circ} 46^{\prime} \mathrm{S}\right.$ e $\left.56^{\circ} 05^{\prime} \mathrm{W}\right)$ está localizada junto ao perímetro urbano de Santo Antônio do Leverger, município situado na extremidade norte do
Pantanal de Poconé (Adámoli 1981). O mapa que localiza a Fazenda Experimental foi adaptado a partir de Amorozo (2002) (figura 1). O entorno da área é formado por vegetação de cerrado (propriedades particulares) e pastagens artificiais (Fazenda Experimental).

A vegetação de cerrado predomina na região (Radambrasil 1982). O clima da região, segundo a classificação de Köppen, é Aw (subtipo savana), com temperaturas altas durante o ano (média anual de $25,6^{\circ} \mathrm{C}$ ), chuvas no verão (outubro-março) e seca no inverno (abril-setembro) (Oliveira Filho 1992). A área estudada pode ser dividida em duas subáreas de acordo com as características do solo durante o período chuvoso. Na sub-área $\mathrm{A}$ (2,7 ha) ocorre saturação de água no solo enquanto na sub-área B (2,2 ha) os solos são bem drenados.

Flora e estrutura $-\mathrm{O}$ levantamento florístico consistiu na coleta mensal de material botânico das espécies arbustivo-arbóreas ao longo de dois anos (1996-1997) em caminhadas aleatórias na área (4,9 ha). As espécies foram identificadas por especialistas e/ou por comparações com amostras de herbário. O material coletado foi herborizado e depositado no Herbário da Universidade Estadual de Campinas - UEC (números de registro: 35416 a $35491 ; 35743$ a 35771; 35828 a 35853 ) e no

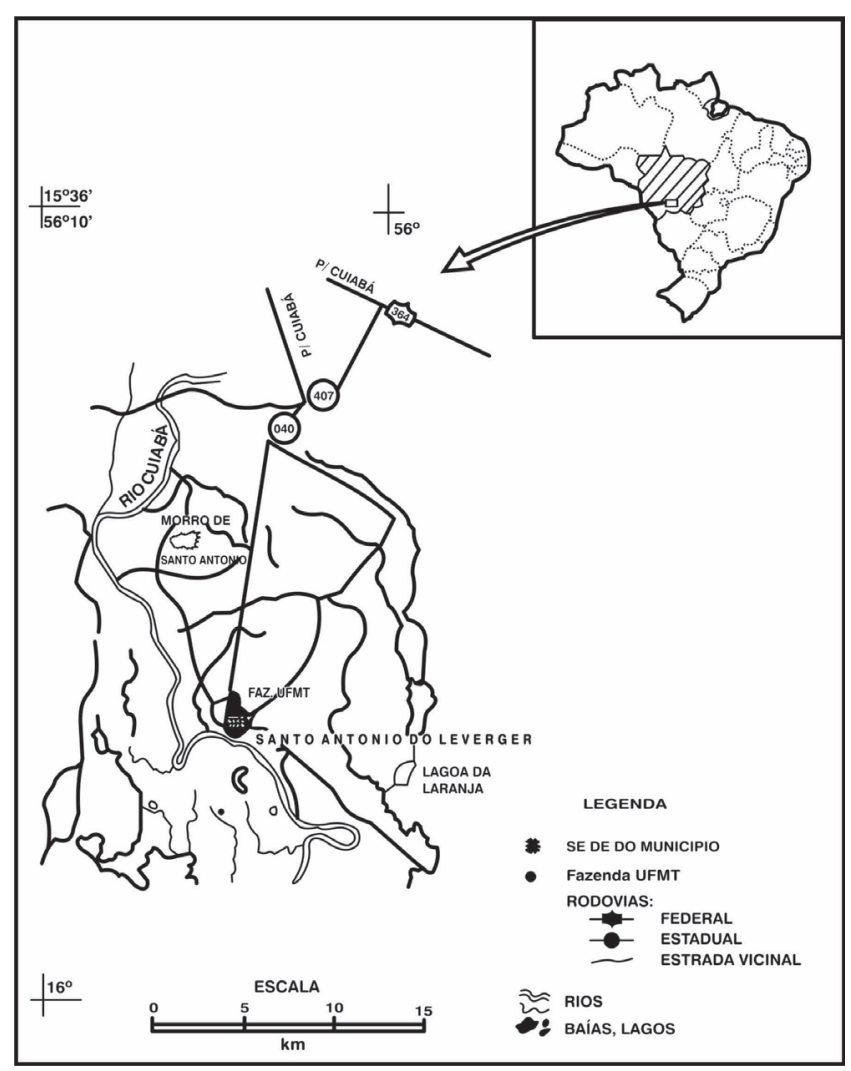

Figura 1. Localização da área de estudo (Fazenda Experimental da UFMT). Fonte: Fundação de Recursos Cândido Rondon.

Figure 1. Localization of study area (Fazenda Experimental da UFMT). Source: Fundação de Recursos Cândido Rondon. 
Herbário Central da Universidade Federal de Mato Grosso $\mathrm{HC}$ (números de registro: 23807 a 23872). As espécies foram ordenadas seguindo o sistema de classificação de Cronquist (1981). A similaridade entre a área estudada e outras com vegetação de cerrado foi estimada através dos índices de Jaccard (IJ) e de Sørensen (IS) (Brower \& Zar 1977). A diversidade florística foi calculada pelo índice de ShannonWeaver, com base em logarítimos naturais (Brower \& Zar 1977).

$$
\mathrm{IJ}=\mathrm{c} /(\mathrm{a}+\mathrm{b}-\mathrm{c}) \quad \mathrm{IS}=2 \mathrm{c} /(\mathrm{a}+\mathrm{b}) \quad \mathrm{H}^{\prime}=-\Sigma \text { pi.ln } \mathrm{pi}
$$

onde, $\quad \mathrm{a}=$ número total de espécies na área $\mathrm{A}$; $\mathrm{b}=$ número total de espécies na área $\mathrm{B} ; \mathrm{c}=$ número de espécies comuns às duas áreas; $\mathrm{pi}=\mathrm{ni} / \mathrm{N} ; \mathrm{ni}=$ número de indivíduos amostrados da espécie $\mathrm{i} ; \mathrm{N}=$ número total de indivíduos amostrados.

A amostragem fitossociológica foi efetuada na sub-área A (2,7 ha) em função da sua maior diversidade florística, conforme observação de campo. A amostragem foi realizada pelo Método de Quadrantes (Cottam \& Curtis 1956), sendo em cada quadrante medida a árvore ou arbusto mais próximo do ponto, totalizando quatro indivíduos em cada ponto. Nas plantas multicaulinares foram medidos todos os caules que formavam um indivíduo. No caso de plantas com protuberâncias na base do caule, as medidas foram realizadas acima do referido espessamento irregular.

Ao longo do maior eixo da área foram instaladas nove trilhas paralelas e eqüidistantes $30 \mathrm{~m}$; as trilhas diferiram no comprimento devido à forma irregular da área. Um total de 62 pontos de coleta, locados a cada $20 \mathrm{~m}$, foi analisado nas trilhas. Outros 38 pontos foram amostrados aleatoriamente, perfazendo 100 pontos de coleta. Foram considerados com hábito arbóreo os indivíduos com caule indiviso, altura $>2 \mathrm{~m}$ e perímetro $\geq 8 \mathrm{~cm}$ (diâmetro $\geq 2,60 \mathrm{~cm}$ ); como arbustivos aqueles com caule indiviso ou ramificado desde a base, altura variando de 0,5 a 2 m e perímetro $\geq 3,5 \mathrm{~cm}$ (diâmetro $\geq 1,11 \mathrm{~cm}$ ). As medidas de perímetro do caule foram tomadas ao nível do solo. As medidas de altura dos indivíduos foram estimadas com uma vara de $3 \mathrm{~m}$, marcada em intervalos de $1 \mathrm{~m}$. Na análise estrutural foram examinados os valores absolutos e relativos de densidade, freqüência e dominância. A soma desses parâmetros, em termos relativos, definiu o índice do valor de importância (IVI) das espécies. As estimativas foram realizadas fazendo uso das fórmulas (Martins 1993): Densidade Total $\left(\mathrm{DTA}=\mathrm{u} / \mathrm{d}^{2}\right)$; Densidade Absoluta $($ DeA $=$ DTA.(ni/N)); Densidade Relativa (DeR = 100.(ni/N); Freqüência Absoluta $(\mathrm{FrA}=100 .(\mathrm{p} / \mathrm{PT}))$; Freqüência Relativa $(\mathrm{FrR}=100 .(\mathrm{FA} / \Sigma \mathrm{FA}))$; Dominância Relativa $(\mathrm{DoR}=100 .(\mathrm{AB} / \Sigma \mathrm{AB}))$; Índice do Valor de Importância $(\mathrm{IVI}=\mathrm{DeR}+\mathrm{FrR}+\mathrm{DoR})$; onde, $\mathrm{u}=$ unidade de área $\left(1\right.$ ha $\left.=10.000 \mathrm{~m}^{2}\right) ; \mathrm{d}^{2}=$ distância média geométrica $(\mathrm{d}=$ antiln $1 / \mathrm{N} .(\ln \mathrm{d} 1+\ln \mathrm{d} 2+\ldots \ln \mathrm{N}))-\mathrm{d} 1, \mathrm{~d} 2, \ldots \mathrm{dN}=$ distâncias individuais, corrigidas com a adição do valor do raio do tronco da planta medida; ni = número de indivíduos amostrados da espécie $\mathrm{i} ; \mathrm{N}=$ número total de indivíduos amostrados; $\mathrm{p}=$ número de pontos amostrados com a espécie $\mathrm{i} ; \mathrm{PT}=$ número total de pontos amostrados; $\mathrm{AB}=$ área basal $\left(\mathrm{AB}=\mathrm{p}^{2} .4 / \pi ; \mathrm{p}=\right.$ perímetro $)$.

\section{Resultados}

Composição Florística - Foram identificadas 131 espécies arbustivo-arbóreas, distribuídas em 92 gêneros e 45 famílias (tabela 1). O hábito de crescimento variou no grau de ocupação do ambiente, com predominância das espécies arbustivas (55\%, $72 \mathrm{spp}$.) sobre as arbóreas (45\%, 59 spp.). As Leguminosae, com 20 táxons, apresentaram maior riqueza florística - Caesalpiniaceae (9 spp.), Fabaceae (9 spp.) e Mimosaceae (2 spp.). Outras famílias comuns na área foram Myrtaceae (10 spp.), Rubiaceae (10 spp.), Annonaceae (8 spp.) e Malpighiaceae (8 spp.).

As famílias com maior número de espécies abrangem 43\% (56/131) dos táxons e 38\% (35/92) dos gêneros encontrados na área. Apenas nove famílias foram representadas por uma espécie. Os gêneros com maior número de espécies foram Annona (5 spp.), Eugenia (4 spp.), Alibertia, Byrsonima, Chomelia, Heteropteys e Qualea reuniram cada uma três espécies. A família mais rica foi Fabaceae, representada por nove espécies de oito gêneros diferentes.

Ao se comparar os dados florísticos da área estudada com outras 20 comunidades de cerrado (sensu lato) (tabela 2) os resultados indicam maior afinidade, em termos florísticos, com o cerrado de Mato Grosso e do Pantanal e menor com outras áreas do planalto central, incluindo aquelas relativamente próximas (ver referências tabela 2). A medida de diversidade específica usada na área estudada, o índice de Shannon-Weaver, alcançou o valor de $\mathrm{H}^{\prime}=3,75$ nats.indivíduo ${ }^{-1}$.

No levantamento florístico foram encontradas 21 espécies características de áreas com cerradão mesotrófico (sensu Ratter 1971) (ver tabela 1).

Estrutura - No levantamento fitossociológico foram amostrados 400 indivíduos lenhosos representando 90 espécies (tabela 3 ). As espécies foram listadas em ordem decrescente do valor de importância. A densidade total calculada foi de 1.653 indivíduos.ha ${ }^{-1}$.

Apenas cinco espécies têm o IVI igual ou superior a 10 ; para a maioria $(78 \%, 70 \mathrm{spp}$.) a importância é menor que 4. As 10 espécies com valores mais altos de IVI somam 49,4\% $(I V I=147,48)$ do total do índice de valor de importância.

A espécie com maior IVI foi Annona dioica, sendo o valor influenciado pela alta densidade e freqüência dos indivíduos. A segunda espécie mais importante na amostragem foi Curatella americana com o IVI sendo afetado pela densidade, freqüência e, principalmente, dominância. 
Tabela 1. Relação das espécies coletadas no cerrado da Fazenda Experimental da UFMT - 1996/97 (ab = arbusto; abe = arbusto escandente; ar = árvore; * = espécies indicadoras de solos mesotróficos - Ratter et al. 1996, 2003).

Table 1. Relation of species colected from the cerrado of Experimental Farm of UFMT - 1996/97 (ab = shrub; abe = winder; ar $=$ tree; $*$ = indicative species of mesotrophic soil - Ratter et al. 1996, 2003).

\begin{tabular}{|c|c|c|}
\hline Família & Espécie & Hábito \\
\hline \multirow[t]{2}{*}{ ANACARDIACEAE } & 1. Astronium fraxinifolium Schott * & ar \\
\hline & 2. Myracrodruon urundeuva Allemão * & ar \\
\hline \multirow[t]{8}{*}{ ANNONACEAE } & 3. Annona cornifolia A.St.-Hil. & $\mathrm{ab}$ \\
\hline & 4. Annona dioica A.St.-Hil. & $\mathrm{ab}$ \\
\hline & 5. Annona phaeoclados Mart. & $\mathrm{ab}$ \\
\hline & 6. Annona sp.1 & $\mathrm{ab}$ \\
\hline & 7. Annona sp.2 & $\mathrm{ab}$ \\
\hline & 8. Cardiopetalum sp. & $\mathrm{ab}$ \\
\hline & 9. Duguetia furfuracea (A.St.-Hil.) Benth. \& Hook.f. & $a b$ \\
\hline & 10. Xylopia aromatica (Lam.) Mart. & $\mathrm{ab}$ \\
\hline \multirow[t]{4}{*}{ APOCYNACEAE } & 11. Aspidosperma cf. subincanum Mart. ex A.DC. * & ar \\
\hline & 12. Hancornia speciosa Gómez. & ar \\
\hline & 13. Himatanthus obovatus (Müll.Arg.) Woodson & ar \\
\hline & 14. Himatanthus sp. & $a b$ \\
\hline \multirow[t]{2}{*}{ ARECACEAE } & 15. Acrocomia aculeata (Jacq.) Lodd. ex Mart. * & ar \\
\hline & 16. Astrocaryum sp. & $a b$ \\
\hline \multirow[t]{2}{*}{ ASTERACEAE } & 17. Vernonia brasiliana Druce & $a b$ \\
\hline & 18. Vernonia ferruginea Less. & $a b$ \\
\hline \multirow[t]{4}{*}{ BIGNONIACEAE } & 19. Jacaranda cuspidifolia Mart. * & ar \\
\hline & 20. Tabebuia aurea (Silva Manso) Benth. \& Hook. f. ex S.Moore & ar \\
\hline & 21. Tabebuia impetiginosa (Mart. ex DC.) Standl. * & ar \\
\hline & 22. Tabebuia sp. & ar \\
\hline \multirow[t]{3}{*}{ BOMBACACEAE } & 23. Pseudobombax longiflorum (Mart. \& Zucc.) A.Robyns & ar \\
\hline & 24. Pseudobombax marginatum (A.St.-Hil. et al.) A.Robyns & $\mathrm{ab}$ \\
\hline & 25. Pseudobombax sp. & ar \\
\hline \multirow[t]{2}{*}{ BORAGINACEAE } & 26. Cordia glabrata (Mart.) A.DC. * & ar \\
\hline & 27. Cordia insignis Cham. & $\mathrm{ab}$ \\
\hline \multirow[t]{9}{*}{ CAESALPINIACEAE } & 28. Bauhinia cf. subclavata Benth. & $\mathrm{ab}$ \\
\hline & 29. Bauhinia sp.1 & $\mathrm{ab}$ \\
\hline & 30. Bauhinia sp.2 & $\mathrm{ab}$ \\
\hline & 31. Copaifera martii Hayne & $a b$ \\
\hline & 32. Copaifera langsdorffii Desf. & ar \\
\hline & 33. Dimorphandra mollis Benth. & ar \\
\hline & 34. Hymenaea stigonocarpa Mart. ex Hayne & ar \\
\hline & 35. Sclerolobium aureum (Tul.) Benth. & ar \\
\hline & 36. Senna chrysocarpa Desv. & $a b$ \\
\hline CARYOCARACEAE & 37. Caryocar brasiliense Cambess. & ar \\
\hline CECROPIACEAE & 38. Cecropia cf. pachystachya Trécul & ar \\
\hline \multirow[t]{2}{*}{ CLUSIACEAE } & 39. Kielmeyera rubriflora Cambess. & $a b$ \\
\hline & 40. Kielmeyera $\mathrm{sp}$ & $a b$ \\
\hline COCHLOSPERMACEAE & 41. Cochlospermum regium (Schrank) Pilg. & $a b$ \\
\hline \multirow[t]{3}{*}{ COMBRETACEAE } & 42. Buchenavia tomentosa (Mart.) Eichler & ar \\
\hline & 43. Combretum discolor Taub. & $a b$ \\
\hline & 44. Terminalia argentea Mart. * & ar \\
\hline CONNARACEAE & 45. Connarus suberosus Planch. & ar \\
\hline \multirow[t]{2}{*}{ DILLENIACEAE } & 46. Curatella americana $\mathrm{L}$. & ar \\
\hline & 47. Davilla elliptica A.St.-Hil. & $a b$ \\
\hline
\end{tabular}


continuação

Família

Espécie

Hábito

EBENACEAE

ERYTHROXYLACEAE

EUPHORBIACEAE

FABACEAE

FLACOURTIACEAE

LAMIACEAE

LYTHRACEAE

MALPIGHIACEAE

MALVACEAE

MELASTOMATACEAE

MIMOSACEAE

MORACEAE

MYRTACEAE

\section{NYCTAGINACEAE \\ OCHNACEAE}

OPILIACEAE
48. Diospyros hispida A.DC.

$\mathrm{ab}$

49. Diospyros sp.

50. Erythroxylum ambiguum Peyr.

$a b$

51. Erythroxylum suberosum A.St.-Hil.

52. Alchornea discolor Endl. \& Poepp.

53. Alchornea sp.

54. Croton cuiabensis Pilg.

55. Acosmium subelegans (Mohlenbr.) Yakovlev

56. Andira cuiabensis Benth.

57. Bowdichia virgilioides Kunth

58. Dipteryx alata Vogel *

59. Luetzelburgia praecox Kuntze

60. Machaerium eriocarpum Benth.

61. Machaerium hirtum (Vell.) Stellfeld

62. Platypodium elegans Vogel *

63. Vatairea cf. macrocarpa (Benth.) Ducke

64. Casearia sylvestris Sw.

65. Hyptis lutescens Pohl ex Benth.

66. Hyptis ovalifolia Benth.

67. Adenaria floribunda Kunth

68. Lafoensia pacari A.St.-Hil.

69. Banisteriopsis pubipetala (A.Juss.) Cuatrec.

70. Byrsonima coccolobifolia Kunth

71. Byrsonima cydoniifolia A.Juss.

72. Byrsonima orbigyniana A.Juss.

73. Heteropteris anoptera A.Juss.

74. Heteropteis aphrodisiaca O. March.

75. Heteropteris sp.

76. Mascagnia benthamiana (Gries.) W.R.Anderson

77. Peltaea speciosa (Kunth) Standley

78. Miconia prasina (Sw.) DC.

79. Rhynchanthera novemnervia DC.

80. Tibouchina aff. karstenii Cogn.

81. Calliandra parviflora Benth. *

82. Stryphnodendron obovatum Benth.

83. Brosimum gaudichaudii Trécul

84. Sorocea saxicola Hassler

85. Campomanesia eugenioides (Cambess.) Legrand

86. Eugenia aurata Berg

87. Eugenia biflora (L.) DC.

88. Eugenia dysenterica Mart. ex DC.*

89. Eugenia sp.

90. Hexachlamys aff. edulis (Berg) Kaus. \& Legrand

91. Myrcia albotomentosa DC.

92. Myrcia tomentosa (Aubl.) DC.

93. Psidium guineense Sw.

94. Psidium sp.

95. Neea theifera Oerst.

96. Ouratea castaneifolia Engl.

97. Ouratea semiserrata (Mart. \& Nees) Engl.

98. Agonandra brasiliensis Miers

\section{$\mathrm{ab}$}

$\mathrm{ab}$

ar

ar

$\mathrm{ab}$

ar

ar

ar

ar

ar

ar

ar

ar

ar

$\mathrm{ab}$

$\mathrm{ab}$

$\mathrm{ab}$

$a b$

ar

abe

ar

ar

$\mathrm{ab}$

$a b$

$a b$

$a b$

abe

$a b$

$a b$

$a b$

$\mathrm{ab}$

$a b$

ar

$a b$

$a b$

$\mathrm{ab}$

$\mathrm{ab}$

$\mathrm{ab}$

ar

ar

$\mathrm{ab}$

$a b$

$a b$

$a b$

$a b$

$a b$

ar

$a b$

ar 
continuação

\begin{tabular}{|c|c|c|}
\hline Família & Espécie & Hábito \\
\hline POLYGONACEAE & 99. Coccoloba mollis Casar. & ar \\
\hline PROTEACEAE & 100. Roupala montana Aubl. & ar \\
\hline RHAMNACEAE & 101. Rhamnidium elaeocarpum Reissek * & ar \\
\hline \multirow[t]{10}{*}{ RUBIACEAE } & 102. Alibertia edulis (L.C.Rich.) A.Rich. & $\mathrm{ab}$ \\
\hline & 103. Alibertia sessilis (Vell.) K.Schum. & $\mathrm{ab}$ \\
\hline & 104. Alibertia verrucosa Moore & $\mathrm{ab}$ \\
\hline & 105. Chomelia obtusa Cham. \& Schltdl. & $\mathrm{ab}$ \\
\hline & 106. Chomelia pohliana Müll.Arg. & $\mathrm{ab}$ \\
\hline & 107. Chomelia sp. & $a b$ \\
\hline & 108. Guettarda viburnoides Cham. \& Schltdl.* & $\mathrm{ab}$ \\
\hline & 109. Randia armata (Sw.) DC. & $\mathrm{ab}$ \\
\hline & 110. Rudgea viburnoides Benth. & $\mathrm{ab}$ \\
\hline & 111. Tocoyena formosa (Cham. \& Schltdl.) K.Schum. & $\mathrm{ab}$ \\
\hline \multirow[t]{2}{*}{ RUTACEAE } & 112. Zanthoxylum hasslerianum (Chodat) Pirani & $\mathrm{ab}$ \\
\hline & 113. Zanthoxylum riedelianum Engl. * & ar \\
\hline \multirow[t]{3}{*}{ SAPINDACEAE } & 114. Dilodendron bipinnatum Raldk. * & ar \\
\hline & 115. Magonia pubescens A.St.-Hil. * & ar \\
\hline & 116. Matayba guianensis Aubl. & ar \\
\hline \multirow[t]{3}{*}{ SAPOTACEAE } & 117. Chrysophyllum marginatum (Hook. \& Arn.) Radlk. & ar \\
\hline & 118. Pouteria ramiflora (Mart.) Raldk. & ar \\
\hline & 119. Pouteria sp. & $\mathrm{ab}$ \\
\hline SIMAROUBACEAE & 120. Simaruba versicolor A.St.-Hil. & ar \\
\hline \multirow[t]{3}{*}{ STERCULIACEAE } & 121. Byttneria cf. filipes Mart. ex Schum. & $\mathrm{ab}$ \\
\hline & 122. Guazuma ulmifolia Lam. * & ar \\
\hline & 123. Helicteres sacarrolha A.St.-Hil. & $\mathrm{ab}$ \\
\hline \multirow[t]{2}{*}{ TILIACEAE } & 124. Luehea cf. candicans Mart.* & ar \\
\hline & 125. Luehea paniculata Mart. * & ar \\
\hline ULMACEAE & 126. Celtis pubescens (H.B.K.) Spreng. & $a b$ \\
\hline VERBENACEAE & 127. Lippia salviaefolia Cham. & $a b$ \\
\hline \multirow[t]{4}{*}{ VOCHYSIACEAE } & 128. Callisthene fasciculata Mart. * & ar \\
\hline & 129. Qualea grandiflora Mart. & ar \\
\hline & 130. Qualea multiflora Mart. & ar \\
\hline & 131. Qualea parviflora Mart. & ar \\
\hline
\end{tabular}

Além de Annona dioica (297) e Curatella americana (95), as espécies mais abundantes foram Peltaea speciosa (95), Hyptis lutescens (78), Vernonia brasiliana (58), Annona cornifolia (45), Byrsonima cydoniifolia (45), Eugenia biflora (45) e Cordia insignis (41). Todas apresentaram 10 ou mais indivíduos amostrados, resultando em maior densidade absoluta por hectare - igual ou superior a 41 indivíduos. Essas nove espécies somam 48\% (799/1653) da densidade total. Em aproximadamente um terço das espécies amostradas (29 spp.) apenas um indivíduo foi computado no levantamento, representando a menor densidade calculada na área (4 indivíduos.ha- ${ }^{-1}$ ).

Quanto à freqüência predominaram valores baixos, com 92\% (83/90) das espécies amostradas exibindo freqüência absoluta menor que $10 \%$. As espécies Annona dioica, Peltaea speciosa e Hyptis lutescens apresentaram os valores mais altos, indicando que as mesmas foram registradas em boa parte dos pontos amostrados (15\% a 46\%). A dominância relativa foi significativa para Curatella americana, Hymenaea stigonocarpa e Byrsonima cydoniifolia, enquanto os demais táxons amostrados apresentaram baixos índices.

$\mathrm{Na}$ distribuição de IVI por família, foram oito as mais importantes, somando $60 \%(\mathrm{IVI}=179,24)$ do valor total: Annonaceae ( $7 \mathrm{spp}$; IVI $=46,29)$, Dilleniaceae (2 spp; IVI $=33,12)$, Caesalpiniaceae (5 spp.; IVI $=22,26)$, Rubiaceae (9 spp.; IVI $=19,62)$, Malpighiaceae (6 spp.; IVI = 18,93), Myrtaceae (7 spp.; IVI $=15,88)$, Vochysiaceae (4 spp.; IVI $=12,77) \mathrm{e}$ 
Tabela 2. Relação de similaridade entre o cerrado da Fazenda Experimental da UFMT (131 spp.) e outras áreas do cerrado (IJ = índice de Jaccard; IS = índice de Sørensen; * = áreas de cerrado distantes cerca de $30 \mathrm{~km}$; número de espécies = número em comum/número total; $\mathrm{MT}=$ Mato Grosso; $\mathrm{DF}=$ Distrito Federal; $\mathrm{GO}=$ Goiás; $\mathrm{MG}=$ Minas Gerais; $\mathrm{MS}=\mathrm{Mato}$ Grosso do Sul; SP = São Paulo.

Table 2. Relation of similarity between of the Experimental Farm da UFMT and other areas of cerrado (IJ = index of Jaccard; IS = index of Sørensen); cerrado area distant around $30 \mathrm{~km}$; number of species = commum number/total number; MT = Mato Grosso; DF = Distrito Federal; GO = Goiás; MG = Minas Gerais; MS = Mato Grosso do Sul; SP = São Paulo.

\begin{tabular}{|c|c|c|c|c|}
\hline Áreas & $\begin{array}{l}\text { Número de } \\
\text { espécies }\end{array}$ & $\begin{array}{l}\mathrm{IJ} \\
(\%)\end{array}$ & $\begin{array}{l}\text { IS } \\
(\%)\end{array}$ & Referências \\
\hline \multicolumn{5}{|l|}{ MT } \\
\hline cerrado & $37 / 111$ & 17,3 & 29,5 & Oliveira Filho \& Martins 1986 \\
\hline cerrado & $26 / 70$ & 25,9 & 14,8 & Oliveira Filho et al. 1989 \\
\hline cerrado* & $22 / 39$ & 14,0 & 25,0 & Nascimento \& Saddi 1992 \\
\hline cerrado* & $21 / 26$ & 15,5 & 27,0 & Guarim Neto et al. 1994 \\
\hline \multicolumn{5}{|l|}{$\mathrm{DF}, \mathrm{GO}, \mathrm{MG}$} \\
\hline cerrado & $15 / 55$ & 8,8 & 16,1 & Ribeiro et al. 1985 \\
\hline cerradão & $19 / 82$ & 9,8 & 17,8 & Ribeiro et al. 1985 \\
\hline cerrado/cerradão & $41 / 122$ & 19,3 & 32,4 & Ratter 1987 \\
\hline cerrado & $13 / 50$ & 7,7 & 8,5 & Oliveira 1991 \\
\hline cerrado & $13 / 50$ & 7,7 & 8,5 & Andrade et al. 2002 \\
\hline cerrado & $21 / 67$ & 11,8 & 21,2 & Silva et al. 2002 \\
\hline \multicolumn{5}{|l|}{ PANTANAL } \\
\hline cerrado - MS & $12 / 22$ & 8,5 & 15,5 & Prance \& Schaller 1982 \\
\hline cerrado - MS & $27 / 56$ & 17,0 & 29,0 & Ratter et al. 1988 \\
\hline cerradão - MS & $35 / 65$ & 21,7 & 35,7 & Ratter et al. 1988 \\
\hline cerradão - MT & $18 / 28$ & 12,8 & 22,6 & Ratter et al. 1988 \\
\hline cerradão - MT & $19 / 33$ & 13,0 & 23,2 & Guarim et al. 2002 \\
\hline \multicolumn{5}{|l|}{ SP } \\
\hline cerrado & $22 / 54$ & 13,5 & 23,8 & Silberbauer-Gottsberger \& Eiten 1983 \\
\hline cerrado & $24 / 101$ & 11,5 & 20,7 & Cesar et al. 1988 \\
\hline cerradão & $25 / 103$ & 12,0 & 21,0 & Toledo Filho et al. 1989 \\
\hline cerrado & $42 / 130$ & 19,2 & 32,2 & Batalha \& Mantovani 2001 \\
\hline cerrado & $17 / 78$ & 8,8 & 16,2 & Durigan et al. 2002 \\
\hline
\end{tabular}

Fabaceae (5 spp.; IVI = 10,37). Annonaceae foi a família mais representativa em função do grande número de indivíduos por hectare e maior freqüência, com destaque para Annona dioica. A menor dominância na família surge como conseqüência do hábito arbustivo das sete espécies amostradas. Dilleniaceae e Caesalpiniaceae apresentaram, inversamente, menor densidade populacional e freqüência e maior biomassa. Nessas duas famílias o valor de dominância foi influenciado pelo hábito arbóreo das espécies amostradas, com exceção de Davilla elliptica (Dilleniaceae) que é arbustiva. As outras famílias apresentaram em comum baixa densidade, freqüência e dominância, indicando que as espécies amostradas são raras e ocupam menor espaço no ambiente.

Perturbações Ambientais - A área estudada está exposta à ação de diferentes agentes de perturbação que podem afetar a composição florística e a estrutura da comunidade: fogo (estação seca), escavações no solo por coletores de minhocuçu (Glossoscolex sp., Oligochaeta) (estação chuvosa), pastejo (contínua) e invasão de espécies não nativas (contínua). Aárea ficou protegida de queimadas por cinco anos (1993-1997).

Nos trabalhos de campo observou-se que durante o pastejo os animais incluem na dieta folhas novas, plântulas, ramos com flores e frutos. Na coleta dos minhocuçus o solo é revolvido em diferentes profundidades e extensão (de 1 a $10 \mathrm{~m}^{2}$ ), eliminando a vegetação do local. Os pastos artificiais, localizados junto a um dos lados da área, facilitam a entrada de espécies invasoras (sensu Pott \& Pott 1994) - Mimosa adenocarpa Benth. e Mimosa debilis Hook \& Arn. 
Tabela 3. Relação das espécies amostradas e seus parâmetros fitossociológicos, ordenadas por valor decrescente de IVI (N. Ind. = número de indivíduos; DeA = densidade absoluta; $D e R=$ densidade relativa; FeA = freqüência absoluta; Fer = freqüência relativa; DoR = dominância relativa; IVI = índice do valor de importância).

Table 3. Relation of species sampled and theirs phytosociological parameters ordered by decreasing value of IVI (N. Ind. = individual numbers; DeA = absolute density; DeR = relative density; FeA = absolute frequency; FeR = relative frequency; DoR = relative dominance; IVI = index of importancy value)

\begin{tabular}{|c|c|c|c|c|c|c|c|c|}
\hline Espécie & $\begin{array}{l}\mathrm{N} . \\
\text { Ind. }\end{array}$ & $\begin{array}{c}\text { N. } \\
\text { Pontos }\end{array}$ & $\begin{array}{c}\text { DeA } \\
\left(\text { Ind.ha }{ }^{-1}\right)\end{array}$ & $\begin{array}{l}\text { DeR } \\
(\%)\end{array}$ & $\begin{array}{l}\mathrm{FeA} \\
(\%)\end{array}$ & $\begin{array}{l}\text { FeR } \\
(\%)\end{array}$ & $\begin{array}{l}\text { DoR } \\
(\%)\end{array}$ & IVI \\
\hline 1. Annona dioica & 72 & 46 & 297 & 18,00 & 46,00 & 14,00 & 4,08 & 36,10 \\
\hline 2. Curatella americana & 23 & 18 & 95 & 5,75 & 18,00 & 5,50 & 21,30 & 32,55 \\
\hline 3. Byrsonima cydoniifolia & 11 & 10 & 45 & 2,75 & 10,00 & 3,00 & 8,18 & 13,93 \\
\hline 4. Hymenaea stigonocarpa & 7 & 7 & 29 & 1,75 & 7,00 & 2,13 & 10,00 & 13,88 \\
\hline 5. Peltaea speciosa & 23 & 15 & 95 & 5,75 & 15,00 & 4,60 & 0,50 & 10,85 \\
\hline 6. Tabebuia aurea & 6 & 6 & 25 & 1,50 & 6,00 & 1,83 & 6,32 & 9,65 \\
\hline 7. Hyptis lutescens & 19 & 15 & 78 & 4,75 & 15,00 & 4,60 & 0,24 & 9,59 \\
\hline 8. Vernonia brasiliana & 14 & 12 & 58 & 3,50 & 12,00 & 3,66 & 0,28 & 7,44 \\
\hline 9. Eugenia biflora & 11 & 10 & 45 & 2,75 & 10,00 & 3,00 & 1,65 & 7,41 \\
\hline 10. Pseudobombax longiflorum & 2 & 2 & 8 & 0,50 & 2,00 & 0,61 & 4,97 & 6,08 \\
\hline 11. Terminalia argentea & 8 & 7 & 33 & 2,00 & 7,00 & 2,13 & 1,66 & 5,80 \\
\hline 12. Annona cornifolia & 11 & 7 & 45 & 2,75 & 7,00 & 2,13 & 0,89 & 5,77 \\
\hline 13. Acrocomia aculeata & 2 & 2 & 8 & 0,50 & 2,00 & 0,61 & 4,58 & 5,69 \\
\hline 14. Cordia insignis & 10 & 9 & 41 & 2,50 & 9,00 & 2,74 & 0,14 & 5,38 \\
\hline 15. Magonia pubescens & 7 & 6 & 29 & 1,75 & 6,00 & 1,83 & 1,80 & 5,38 \\
\hline 16. Qualea grandiflora & 6 & 6 & 25 & 1,50 & 6,00 & 1,83 & 1,96 & 5,29 \\
\hline 17. Astronium fraxinifolium & 6 & 6 & 25 & 1,50 & 6,00 & 1,83 & 1,94 & 5,27 \\
\hline 18. Alibertia edulis & 6 & 6 & 25 & 1,50 & 6,00 & 1,83 & 1,50 & 4,83 \\
\hline 19. Qualea parviflora & 6 & 6 & 25 & 1,50 & 6,00 & 1,83 & 0,98 & 4,31 \\
\hline 20. Simarouba versicolor & 3 & 3 & 12 & 0,75 & 3,00 & 0,91 & 2,45 & 4,11 \\
\hline 21. Croton cuiabensis & 7 & 7 & 29 & 1,75 & 7,00 & 2,13 & 0,05 & 3,93 \\
\hline 22. Sclerolobium aureum & 2 & 2 & 8 & 0,50 & 2,00 & 0,61 & 2,52 & 3,63 \\
\hline 23. Chomelia pohliana & 7 & 5 & 29 & 1,75 & 5,00 & 1,52 & 0,18 & 3,45 \\
\hline 24. Chomelia obtusa & 5 & 4 & 20 & 1,25 & 4,00 & 1,22 & 0,70 & 3,20 \\
\hline 25. Luetzelburgia praecox & 2 & 2 & 8 & 0,50 & 2,00 & 0,61 & 1,61 & 2,72 \\
\hline 26. Dilodendron bipinnatum & 2 & 2 & 8 & 0,50 & 2,00 & 0,61 & 1,50 & 2,61 \\
\hline 27. Combretum discolor & 4 & 4 & 16 & 1,00 & 4,00 & 1,22 & 0,17 & 2,39 \\
\hline 28. Pouteria ramiflora & 1 & 1 & 4 & 0,25 & 1,00 & 0,30 & 1,84 & 2,39 \\
\hline 29. Machaerium eriocarpum & 4 & 3 & 16 & 1,00 & 3,00 & 0,91 & 0,45 & 2,36 \\
\hline 30. Andira cuiabensis & 2 & 2 & 8 & 0,50 & 2,00 & 0,61 & 1,21 & 2,32 \\
\hline 31. Helicteres sacarrolha & 4 & 4 & 16 & 1,00 & 4,00 & 1,22 & 0,09 & 2,31 \\
\hline 32. Erythroxylum ambiguum & 4 & 3 & 16 & 1,00 & 3,00 & 0,91 & 0,22 & 2,13 \\
\hline 33. Caryocar brasiliense & 1 & 1 & 4 & 0,25 & 1,00 & 0,30 & 1,53 & 2,08 \\
\hline 34. Campomanesia eugenioides & 3 & 3 & 12 & 0,75 & 3,00 & 0,91 & 0,37 & 2,03 \\
\hline 35. Tocoyena formosa & 3 & 3 & 12 & 0,75 & 3,00 & 0,91 & 0,30 & 1,96 \\
\hline 36. Sorocea saxicola & 3 & 3 & 12 & 0,75 & 3,00 & 0,91 & 0,22 & 1,88 \\
\hline 37. Copaifera martii & 3 & 3 & 12 & 0,75 & 3,00 & 0,91 & 0,12 & 1,78 \\
\hline 38. Heteropteris anoptera & 3 & 3 & 12 & 0,75 & 3,00 & 0,91 & 0,10 & 1,76 \\
\hline 39. Lafoensia pacari & 2 & 2 & 8 & 0,50 & 2,00 & 0,61 & 0,65 & 1,76 \\
\hline 40. Zanthoxylum riedelianum & 2 & 2 & 8 & 0,50 & 2,00 & 0,61 & 0,65 & 1,76 \\
\hline 41. Callisthene fasciculata & 2 & 1 & 8 & 0,50 & 1,00 & 0,30 & 0,95 & 1,75 \\
\hline 42. Eugenia aurata & 3 & 3 & 12 & 0,75 & 3,00 & 0,91 & 0,08 & 1,74 \\
\hline 43. Senna chrysocarpa & 3 & 3 & 12 & 0,75 & 3,00 & 0,91 & 0,07 & $\begin{array}{c}1,73 \\
\text { ontinua }\end{array}$ \\
\hline
\end{tabular}


continuação

\begin{tabular}{|c|c|c|c|c|c|c|c|c|}
\hline Espécie & $\begin{array}{l}\mathrm{N} . \\
\text { Ind. }\end{array}$ & $\begin{array}{c}\text { N. } \\
\text { Pontos }\end{array}$ & $\begin{array}{c}\text { DeA } \\
\left(\text { Ind.ha' }{ }^{-1}\right)\end{array}$ & $\begin{array}{l}\text { DeR } \\
(\%)\end{array}$ & $\begin{array}{c}\mathrm{FeA} \\
(\%)\end{array}$ & $\begin{array}{l}\text { FeR } \\
(\%)\end{array}$ & $\begin{array}{c}\text { DoR } \\
(\%)\end{array}$ & IVI \\
\hline 44. Tibouchina aff. karstenii & 3 & 2 & 12 & 0,75 & 2,00 & 0,61 & 0,27 & 1,63 \\
\hline 45. Myrcia albotomentosa & 3 & 2 & 12 & 0,75 & 2,00 & 0,61 & 0,19 & 1,55 \\
\hline 46. Guettarda viburnoides & 3 & 2 & 12 & 0,75 & 2,00 & 0,61 & 0,15 & 1,51 \\
\hline 47. Qualea multiflora & 2 & 2 & 8 & 0,50 & 2,00 & 0,61 & 0,31 & 1,42 \\
\hline 48. Aspidosperma cf. subincanum & 1 & 1 & 4 & 0,25 & 1,00 & 0,30 & 0,86 & 1,41 \\
\hline 49. Brosimum gaudichaudii & 3 & 2 & 12 & 0,75 & 2,00 & 0,61 & 0,05 & 1,41 \\
\hline 50. Buchenavia tomentosa & 2 & 2 & 8 & 0,50 & 2,00 & 0,61 & 0,26 & 1,37 \\
\hline 51. Duguetia furfuracea & 2 & 2 & 8 & 0,50 & 2,00 & 0,61 & 0,25 & 1,36 \\
\hline 52. Randia armata & 2 & 2 & 8 & 0,50 & 2,00 & 0,61 & 0,09 & 1,20 \\
\hline 53. Acosmium subelegans & 1 & 1 & 4 & 0,25 & 1,00 & 0,30 & 0,64 & 1,19 \\
\hline 54. Rudgea viburnoides & 2 & 2 & 8 & 0,50 & 2,00 & 0,61 & 0,08 & 1,19 \\
\hline 55. Cardiopetalum sp. & 2 & 2 & 8 & 0,50 & 2,00 & 0,61 & 0,07 & 1,18 \\
\hline 56. Psidium guineense & 2 & 2 & 8 & 0,50 & 2,00 & 0,61 & 0,07 & 1,18 \\
\hline 57. Chomelia sp. & 2 & 2 & 8 & 0,50 & 2,00 & 0,61 & 0,05 & 1,16 \\
\hline 58. Myrcia tomentosa & 2 & 2 & 8 & 0,50 & 2,00 & 0,61 & 0,05 & 1,16 \\
\hline 59. Calliandra parviflora & 2 & 2 & 8 & 0,50 & 2,00 & 0,61 & 0,04 & 1,15 \\
\hline 60. Heteropteris aphrodisiaca & 1 & 1 & 4 & 0,25 & 1,00 & 0,30 & 0,02 & 1,13 \\
\hline 61. Alibertia verrucosa & 1 & 1 & 4 & 0,25 & 1,00 & 0,30 & 0,57 & 1,12 \\
\hline 62. Cochlospermum regium & 2 & 2 & 8 & 0,50 & 2,00 & 0,61 & 0,01 & 1,12 \\
\hline 63. Rhynchanthera novemnervia & 2 & 2 & 8 & 0,50 & 2,00 & 0,61 & 0,01 & 1,12 \\
\hline 64. Luehea paniculata & 2 & 2 & 8 & 0,50 & 2,00 & 0,61 & 0,54 & 1,09 \\
\hline 65. Xylopia aromatica & 1 & 1 & 4 & 0,25 & 1,00 & 0,30 & 0,53 & 1,08 \\
\hline 66. Platypodium elegans & 1 & 1 & 4 & 0,25 & 1,00 & 0,30 & 0,46 & 1,01 \\
\hline 67. Alchornea discolor & 1 & 1 & 4 & 0,25 & 1,00 & 0,30 & 0,43 & 0,98 \\
\hline 68. Hyptis ovalifolia & 2 & 1 & 8 & 0,50 & 1,00 & 0,30 & 0,16 & 0,96 \\
\hline 69. Cecropia cf. pachystachya & 1 & 1 & 4 & 0,25 & 1,00 & 0,30 & 0,36 & 0,91 \\
\hline 70. Coccoloba mollis & 1 & 1 & 4 & 0,25 & 1,00 & 0,30 & 0,36 & 0,91 \\
\hline 71. Guazuma ulmifolia & 1 & 1 & 4 & 0,25 & 1,00 & 0,30 & 0,36 & 0,91 \\
\hline 72. Mascagnia benthamiana & 2 & 1 & 8 & 0,50 & 1,00 & 0,30 & 0,06 & 0,86 \\
\hline 73. Ouratea castaneifolia & 1 & 1 & 4 & 0,25 & 1,00 & 0,30 & 0,30 & 0,85 \\
\hline 74. Hexachlamys aff. edulis & 2 & 1 & 8 & 0,50 & 1,00 & 0,30 & 0,01 & 0,81 \\
\hline 75. Dipteryx alata & 1 & 1 & 4 & 0,25 & 1,00 & 0,30 & 0,22 & 0,77 \\
\hline 76. Rhamnidium elaeocarpum & 1 & 1 & 4 & 0,25 & 1,00 & 0,30 & 0,19 & 0,74 \\
\hline 77. Annona sp.1 & 1 & 1 & 4 & 0,25 & 1,00 & 0,30 & 0,14 & 0,69 \\
\hline 78. Bauhinia sp.2 & 1 & 1 & 4 & 0,25 & 1,00 & 0,30 & 0,13 & 0,68 \\
\hline 79. Cordia glabrata & 1 & 1 & 4 & 0,25 & 1,00 & 0,30 & 0,12 & 0,67 \\
\hline 80. Agonandra brasiliensis & 1 & 1 & 4 & 0,25 & 1,00 & 0,30 & 0,11 & 0,66 \\
\hline 81. Byrsonima coccolobifolia & 1 & 1 & 4 & 0,25 & 1,00 & 0,30 & 0,09 & 0,64 \\
\hline 82. Chrysophyllum marginatum & 1 & 1 & 4 & 0,25 & 1,00 & 0,30 & 0,09 & 0,64 \\
\hline 83. Heteropteris sp. & 1 & 1 & 4 & 0,25 & 1,00 & 0,30 & 0,06 & 0,61 \\
\hline 84. Erythroxylum suberosum & 1 & 1 & 4 & 0,25 & 1,00 & 0,30 & 0,05 & 0,60 \\
\hline 85. Celtis pubescens & 1 & 1 & 4 & 0,25 & 1,00 & 0,30 & 0,03 & 0,58 \\
\hline 86. Davilla elliptica & 1 & 1 & 4 & 0,25 & 1,00 & 0,30 & 0,02 & 0,57 \\
\hline 87. Bauhinia sp.1 & 1 & 1 & 4 & 0,25 & 1,00 & 0,30 & 0,01 & 0,56 \\
\hline 88. Ouratea semiserrata & 1 & 1 & 4 & 0,25 & 1,00 & 0,30 & 0,01 & 0,56 \\
\hline 89. Pseudobombax marginatum & 1 & 1 & 4 & 0,25 & 1,00 & 0,30 & 0,01 & 0,56 \\
\hline 90. Annona phaeoclados & 1 & 1 & 4 & 0,25 & 1,00 & 0,30 & 0,30 & 0,11 \\
\hline Total & 400 & 100 & 1653 & 100,00 & 100,00 & 100,00 & 100,00 & 298,54 \\
\hline
\end{tabular}


(Mimosaceae) e Melinis minutiflora Beauv. (capimgordura, Poaceae). Essas espécies têm comportamento agressivo principalmente o capim-gordura, que exibe dominância em alguns espaços da área sobre as espécies nativas.

\section{Discussão}

O levantamento florístico revelou significativa riqueza de espécies arbustivo-arbóreas na área estudada (131 spp.). O número de espécies foi influenciado diretamente pela inclusão de arbustos finos no levantamento. Em função da adoção desse critério contribuíram para a riqueza florística Annona dioica, A. phaeoclados, Bauhinia sp.1, Brosimum gaudichaudii, Croton cuiabensis, Heteropteris aphrodisiaca, Hexaclamys aff. edulis, Hyptis lutescens, Lippia salviaefolia, Peltaea speciosa, Pseudobombax marginatum, Rhinchanthera novemnervia e Vernonia ferruginea.

A definição do hábito arbustivo na vegetação do cerrado muitas vezes é subjetiva. Algumas espécies listadas no presente levantamento foram citadas como herbáceas (Batalha \& Mantovani 2001, Weiser \& Godoy 2001) ou como arbustivas (Mendonça et al. 1998) em outros estudos. Esses dados ilustram a discussão de Castro et al. (1999), sobre a dificuldade de se definir uma espécie "lenhosa" no cerrado. Para os autores tal dificuldade seria conseqüência da não existência de critério que possa ser usado universalmente, ou porque pesquisadores diferem no conceito que utilizam e porque algumas espécies exibem variações no hábito de crescimento entre localidades, desde herbáceo até arbóreo. Um exemplo é oferecido por B. gaudichaudii, que na área estudada cresce como arbusto fino e em cerrado de São Paulo o diâmetro dos indivíduos é superior a $3 \mathrm{~cm}$, segundo Cesar et al. (1988).

No cerrado, além do clima, do substrato e das características genéticas, outros fatores como o fogo (Coutinho 1980, Raw \& Hay 1985) e a poda das plantas (Ferri 1971), podem afetar o desenvolvimento dos indivíduos, impedindo muitas vezes a definição sobre o hábito de crescimento das espécies. Portanto, as considerações acima permitem julgar a composição florística na área estudada como parte da vegetação arbustivo-arbórea do cerrado como um todo.

A maioria das espécies computadas no presente estudo, 68\% (89/131), é encontrada em outras áreas com vegetação de cerrado no país. Essas espécies foram citadas por Ratter et al. (2003) na análise florística de 315 áreas de cerrado (excluindo alguns sítios na
Amazônia), englobando 914 espécies lenhosas (diâmetro $\geq 3 \mathrm{~cm}$ ). O estudo revelou que 300 espécies, observadas em oito ou mais áreas, representam as mais comuns, sendo encontradas em pelo menos $75 \%$ do cerrado. Nessa listagem de 300 espécies com ampla distribuição estão incluídas $86(96,6 \%)$ das 89 espécies registradas na área estudada e citadas pelos autores. Apenas três espécies foram indicadas pelos mesmos como raras, em função da sua presença em apenas uma localidade, Heteropteris anoptera, Senna chrysocarpa e Vernonia brasiliana.

As famílias que descrevem melhor a flora da área estudada são características da vegetação do cerrado. Castro et al. (1999) relacionaram mais de 150 espécies de Leguminosae (Caesalpiniaceae, Fabaceae e Mimosaceae), 70 de Myrtaceae, 58 de Malpighiaceae, 55 de Rubiaceae e 30 de Annonaceae. Outras famílias encontradas na área também se destacam no cerrado, como Dilleniaceae, Erythroxylaceae e Melastomataceae (Goodland 1970, Goodland \& Ferri 1979), além de Vochysiaceae, que pode ser dominante em muitas localidades (Ratter et al. 1997).

Os gêneros que mais contribuíram com a riqueza específica na área estudada são igualmente importantes no cerrado. Goodland (1970) e Heringer et al. (1977) relacionam Annona, Alibertia, Byrsonima, Eugenia, Heteropteris e Qualea entre os que caracterizam esse tipo de vegetação. Esses gêneros somados a outros 85 conferem riqueza de gêneros na área estudada.

Comparando a área estudada com outras comunidades através dos índices de similaridade de Jaccard (IJ) e de Sørensen (IS), ficou clara a variação na composição florística entre locais, sustentando o padrão já detectado para o bioma cerrado (Felfili \& Silva Júnior 1993, Ratter et al. 1996, 1997 e 2003). A afinidade florística foi maior com o cerrado (sensu lato) de Mato Grosso e do Pantanal. Entretanto, essa afinidade é baixa se for considerado apenas o índice de Jaccard (IJ). Müller-Dombois \& Ellenberg (1974) sugerem que, com o uso desse índice, para que ocorra similaridade florística pelo menos $25 \%$ das espécies devem ser comuns entre duas comunidades. A divergência nos resultados provavelmente se deva a variação no esforço de amostragem e no diâmetro mínimo de inclusão dos indivíduos adotados nos estudos. Esses dois fatores influenciam diretamente na inclusão ou exclusão de espécies nos levantamentos florísticos.

Além da riqueza de espécies outra forma de se medir a diversidade de uma comunidade é através do uso de índices, como o de Shannon-Weaver (H'). Na área estudada esse índice alcançou 3,75 nats.indivíduo ${ }^{-1}$. 
O valor obtido quanto ao índice de Shannon-Weaver complementa os dados analisados por Ratter et al. (1997, 2003), que apontam Mato Grosso com um dos estados brasileiros onde a vegetação do cerrado exibe maior diversidade.

A flora arbustivo-arbórea da comunidade estudada é composta por espécies encontradas em áreas de cerrado (sensu stricto) e também táxons característicos de cerradão. $O$ total de 21 espécies listadas na tabela 1 foi citado por Ratter et al. $(1996,2003)$ como típicas de cerradões com solo mesotrófico. A distinção entre cerradão mesotrófico e distrófico foi feita por Ratter (1971), com base na composição florística e propriedades do solo. Segundo o autor um cerradão mesotrófico apresenta solos menos ácidos, com maior quantidade de matéria orgânica e nutrientes e Magonia pubescens (Sapindaceae) é a espécie que melhor define a vegetação. Cerradões mesotróficos são encontrados em Goiás, Mato Grosso, Mato Grosso do Sul e Minas Gerais (Furley \& Ratter 1988), sendo os mesmos usados como parâmetro para se delimitar padrões de composição florística para o cerrado (Ratter et al. 1996, 2003).

As espécies identificadas de cerradão provavelmente se estabeleceram na área estudada a partir de sementes provenientes desta fisionomia de vegetação que ainda faz parte da vegetação do cerrado na região.

Os resultados obtidos no levantamento florístico apontando expressiva riqueza de espécies e de gêneros, baixa similaridade com outros locais que apresentam vegetação de cerrado, alto valor do índice de diversidade e presença de táxons que caracterizam flora de cerradão indicam que a área estudada serve como exemplo de heterogeneidade da vegetação do cerrado (alfa e beta diversidade). De acordo com Ratter et al. (1997) e Furley (1999) a expressiva heterogeneidade florística do cerrado deve ser considerada em programas de conservação, sendo necessário proteger muitas áreas para conseguir preservar toda a sua biodiversidade.

$\mathrm{O}$ estudo fitossociológico permitiu caracterizar a estrutura do estrato arbustivo-arbóreo através da estimativa de parâmetros relacionados com as populações das espécies. O número estimado de 1.653 indivíduos.ha ${ }^{-1}$ sugere que o cerrado estudado é relativamente denso.

$\mathrm{Na}$ área estudada, entre as espécies arbustivas, como Annona dioica, a densidade e freqüência foram determinantes na definição do IVI. Para as arbóreas, como Curatella americana, foram a densidade e dominância os parâmetros mais importantes. $\mathrm{Na}$ amostragem espécies com maior densidade absoluta exibiram geralmente IVI mais significativo. Aproximadamente um terço das espécies exibiu baixa densidade absoluta ( 4 indivíduos.ha ${ }^{-1}$ ). Baixa densidade pode afetar o sucesso reprodutivo das espécies e, por conseqüência, a manutenção de populações no ambiente. Isso porque caso a população seja pequena maior é a probabilidade de cruzamento entre indivíduos aparentados, levando à endogamia, nas espécies com fecundação cruzada (Richards 1986). Esse é o sistema reprodutivo observado em várias espécies arbustivoarbóreas da vegetação do cerrado, que é definido pela presença de auto-incompatibilidade genética e dioicia (Oliveira \& Gibbs 2000). Sendo assim, a maior exposição de alelos recessivos com a endogamia aumentam as chances de extinção local de populações.

$\mathrm{Na}$ área estudada foi observado que os maiores valores de IVI $(\geq 10)$ estavam restritos a um grupo de cinco espécies. As demais espécies amostradas apresentaram IVI pouco significativo. Isso indica que, para a maioria das espécies, predominam baixa densidade, freqüência e dominância no estrato arbustivoarbóreo. Resultados semelhantes foram registrados em outras áreas de cerrado no país, sendo que a diferença entre áreas está na composição das espécies amostradas (Ribeiro et al. 1985, Cesar et al. 1988, Nascimento \& Saddi 1992, Guarim Neto et al. 1994). A análise de parâmetros fitossociológicos em áreas de cerrado (sensu stricto) revela, portanto, variações locais de estrutura e composição florística.

Em relação às famílias amostradas no estudo sobre a estrutura foi observada dominância de família por Annonaceae, uma vez que reuniu maior número de indivíduos amostrados. A maior densidade implicou em maior soma de IVI para essa família. Em outras áreas de cerrado, em termos de IVI, as famílias Vochysiaceae e Leguminosae (Caesalpiniaceae e Fabaceae) são mais importantes (Ribeiro et al. 1985, Cesar et al. 1988, Nascimento \& Saddi 1992). Na área estudada Vochysiaceae e Fabaceae, entretanto, apresentam pouca significância.

Perturbações ambientais - $\mathrm{O}$ presente trabalho foi desenvolvido em um cerrado ao qual, sob contexto ecológico, não se aplica à idéia de fragmento. A separação da área estudada de outras com igual vegetação é artificialmente criada por uma cerca, não estabelecendo, portanto, isolamento geográfico. Por outro lado, em termos de extensão, a área estudada constitui um fragmento dentro dos limites territoriais da Fazenda Experimental da UFMT, justificando cuidados que permitam a continuidade da comunidade. 
A baixa densidade populacional de muitas espécies gera preocupação quanto à possibilidade de sobrevivência de populações na comunidade. Além disso, dentro da Fazenda Experimental, são mantidos, junto à borda da área trabalhada (4,9 ha), pastos artificiais que promovem o isolamento parcial da mesma em relação à vegetação natural. Isso tem favorecido o estabelecimento de espécies invasoras no cerrado estudado. O pastejo pode comprometer o desenvolvimento dos indivíduos e, também, reduzir as chances de sucesso reprodutivo das espécies cujas flores e frutos imaturos são consumidas neste processo. A coleta de minhocuçus remove a vegetação em vários pontos da área, podendo potencialmente alterar a estrutura da população local das espécies.

As pressões impostas à comunidade pelas perturbações ambientais, incluindo alteração ou destruição da vegetação do entorno, colocam em risco a riqueza florística observada para essa área em particular, justificando medidas de conservação em curto prazo.

Agradecimentos - Aos especialistas que identificaram espécies citadas no presente trabalho - Ângela B. Martins (Unicamp) em Melastomataceae, Carlos Vítor Mendonça Filho (Unicamp) em Machaerium spp., Graziela M. Barroso (Jardim Botânico do Rio de Janeiro) em Myrtaceae, Jorge P.P. Carauta (Feema-RJ) em Moraceae, Kikyo Yamamoto (Unicamp) em Ochnaceae, M. Lúcia Kawasaki (Instituto de Botânica-SP) em Myrtaceae, Maria Cândida H. Mamede (Instituto de Botânica-SP) em Malpighiaceae, e Washington M. Ferraz (Unicamp) em Polygonaceae. Ao Júlio Antônio Ribeiro pela grande ajuda nos trabalhos de campo. À Faculdade de Agronomia e Medicina Veterinária (Famev), da Universidade Federal de Mato Grosso (UFMT), por permitir o desenvolvimento deste trabalho na Fazenda Experimental. Ao Conselho Nacional de Desenvolvimento Científico e Tecnológico $(\mathrm{CNPq})$ pelo apoio financeiro.

\section{Referências bibliográficas}

ADÁMOLI, J. 1981. O Pantanal e suas relações fitogeográficas com os cerrados. In Anais do XXXII Congresso Nacional de Botânica, Sociedade Brasileira de Botânica, Teresina, p.109-119.

AMOROZO, M.C.M. 2002. Uso e diversidade de plantas medicinais em Santo Antônio do Leverger, MT, Brasil. Acta Botanica Brasilica 16:189-203.

ANDRADE, L.A.Z., FELFILI, J.M. \& VIOLATTI, L. 2002. Fitossociologia de uma área de cerrado denso na RECOR-IBGE, Brasília - DF. Acta Botanica Brasilica 16:225-240.
AZEVEDO, L.G, MOREIRA, A.G, BARBOSA,A.S., NELSON, B., RIBEIRO, J.F. \& CORADIN, L. 1995. Vegetação do cerrado. In Conhecimento científico para gestão ambiental: amazônia, cerrado e pantanal. Tomo II. Meio Natural (Ministério do Meio Ambiente, dos Recursos Hídricos e da Amazônia Legal/ Instituto Brasileiro do meio Ambiente e dos Recursos Naturais Renováveis, coord.), IBAMA/DITAN, Brasília, p.527-548.

BATALHA, M.A. \& MANTOVANI, W. 2001. Floristic composition of the cerrado in the Pé-de-Gigante Reserve (Santa Rita do Passa Quatro, southeastern Brazil). Acta Botanica Brasilica 15:289-304.

BRASIL. 1999. Ações Prioritárias para a Conservação da Biodiversidade do Cerrado e Pantanal. Ministério do Meio Ambiente/Funatura/Fundação Biodiversitas/ Universidade de Brasília, Brasília.

BROWER, J.E. \& ZAR, J.H. 1977. Field \& laboratory methods for general ecology. Wm. C. Brown Publishers, Dubuque.

CASTRO, A.A.J.F., MARTINS, F.R., TAMASHIRO, J.Y. \& SHEPHERD, G.J. 1999. How rich is the flora of Brazilian cerrados? Annals of the Missouri Botanical Garden 86:192-224.

CESAR, O., PAGANO, S.N., LEITÃO FILHO, H.F, MONTEIRO, R., SILVA, O.A., MARINIS, G. \& SHEPHERD, G.J. 1988. Estrutura fitossociológica do estrato arbóreo de uma área de vegetação de cerrado no município de Corumbataí (Estado de São Paulo). Naturalia 13:91-101.

COTTAM, G. \& CURTIS, J.T. 1956. The use of distance measures in phytosociological sampling. Ecology 37:451-460.

COUTINHO, L.M. 1978. O conceito de cerrado. Revista Brasileira de Botânica 1:17-23

COUTINHO, L.M. 1980. As queimadas e seu papel ecológico. Brasil Florestal 44:7-23.

CRONQUIST, A. 1981. An integrated system of classification of flowering plants. Columbia University Press, New York.

DURIGAN, G., NISHIKAWA, D.L.L., ROCHA, G., SILVEIRA, E.R., PULITANO, F.M., REGALADO, L.B., CARVALHAES, M.A., PARANAGUÁ, P.A. \& RANIERI, V.E.L. 2002. Caracterização de dois estratos da vegetação em uma área de cerrado no município de Brotas, SP, Brasil. Acta Botanica Brasilica 16:251-262.

EITEN, G. 1994. Vegetação do Cerrado. In Cerrado: Caracterização, ocupação e perspectivas (M.N. Pinto, org.). Editora da Universidade de Brasília - Edunb/ Secretaria do Meio Ambiente, Ciência e Tecnologia Sematec, Brasília, p.17-73.

FELFILI, J.M. \& SILVA JUNIOR, M.C. 1993. A comparative study of cerrado (sensu stricto) vegetation in Central Brazil. Journal of Tropical Ecology 9:277-289.

FERREIRA, M.S.F.D. \& MONTEIRO, J.R.B. 1990. Mato Grosso. Impacto Ambiental na área de abrangência da BR-364. Programa Polonoroeste. SCT/PR-CNPq. Relatório de Pesquisa 10. 
FERRI, M.G. 1971. Modificação do hábito floral de Cochlospermum regium (Mart.) Pilger. In III Simpósio sobre o cerrado (M.G. Ferri, coord.). Editora Edgard Blücher Ltda/ Editora da Universidade de São Paulo Edusp, São Paulo, p.164-166.

FURLEY, P.A. 1999. The nature and diversity of neotropical savanna vegetation with particular reference to the Brazilian cerrados. Global Ecology \& Biogeography 8:223-241.

FURLEY, P.A. \& RATTER, J.A. 1988. The central Brazilian cerrado and their development. Journal of Biogeography 15:97-108.

GOODLAND, R.J.A. 1970. Plants of the cerrado vegetation of Brasil. Phytologia 20:57-78.

GOODLAND, R. \& FERRI, M.G. 1979. Ecologia do Cerrado. Editora da Universidade de São Paulo - Edusp/Livraria Itatiaia Editora Ltda., Belo Horizonte.

GUARIMNETO, G, GUARIM, V.L.M.S. \& PRANCE, GT. 1994. Structure and floristic composition of the trees of an area of cerrado near Cuiabá, Mato Grosso, Brazil. Kew Bulletin 49:499-509.

GUARIM, V.L.M.S., MORAES, E.C.C, PRANCE, G.T. \& RATTER, J.A. 2002. Inventory of a mesotrophic Callisthene cerradão in the Pantanal of Mato Grosso, Brazil. Edinburgh Journal of Botany 57:429-436.

HERINGER, E.P., BARROSO, G.M., RIZZO, J.A. \& RIZZINI, C.T. 1977. A flora do cerrado. In IV Simpósio sobre o Cerrado (M.G. Ferri, coord.). Editora Universidade de São Paulo - Edusp/Livraria Itatiaia Editora Ltda., São Paulo, p.211-232.

MARTINS, F.R. 1993. Estrutura de uma mata mesófila. Editora da Universidade Estadual de Campinas - Unicamp, Campinas.

MENDONÇA, R.C., FELFILI, J.M., WALTER, B.M.T., SILVA JÚNIOR, M.C., REZENDE, A.V., FILGUEIRAS, T.S. \& NOGUEIRA, P.E. 1998. Flora vascular do cerrado. In Cerrado: Ambiente e Flora (S.M. Sano, S.P. Almeida, eds.). Embrapa, Planaltina, p.289-556.

MÜLLER-DOMBOIS, D. \& ELLENBERG, H. 1974. Aims and methods in vegetation ecology. John Wiley and Sons, New York.

NASCIMENTO, M.T. \& SADDI, N. 1992. Structure and floristic composition in an area of cerrado in Cuiabá MT, Brazil. Revista Brasileira de Botânica 15:47-55.

OLIVEIRA, P.E. 1991. The pollination and reproductive biology of a cerrado woody community in Brazil. $\mathrm{PhD}$ thesis, University of St. Andrews, Scotland.

OLIVEIRA, P.E. \& GIBBS, P.E. 2000. Reproductive biology of woody plants in a cerrado community of Central Brazil. Flora 195:311-329.

OLIVEIRA FILHO, A.T. 1992. The vegetation of Brazilian "murundus" - the island-effect on the plant community. Journal of Tropical Ecology 8:465-486.
OLIVEIRAFILHO,A.T. \&. MARTINS, F.R 1986. Distribuição, caracterização e composição florística das formações vegetais da região da Salgadeira, na Chapada dos Guimarães (MT). Revista Brasileira de Botânica 9:207-223.

OLIVEIRAFILHO, A.T., SHEPHERD, GJ., MARTINS, F.R. \& STUBBLEBINE, W.H. 1989. Environmental factors affecting physionomic and floristic variation in an area of cerrado in central Brazil. Journal of Tropical Ecology 5:413-431.

POTT, A. \& POTT, V.J. 1994. Plantas do Pantanal. EmbrapaCPAP, Corumbá.

PRANCE, G.T. \& SCHALLER, G.B. 1982. Preliminary study of some vegetation types of the Pantanal, Mato Grosso, Brazil. Brittonia 34:228-251.

RADAMBRASIL 1982. Levantamento de recursos naturais. V.26. Folha SD.21 Cuiabá. Ministério das Minas e Energia, Rio de Janeiro, p.405-429.

RATTER, J.A 1971. Some notes on two types of cerradão occurring in north eastern Mato Grosso. In III Simpósio sobre o cerrado (M.G. Ferri, coord.). Editora Edgard Blücher Ltda/ Editora da Universidade de São Paulo Edusp, São Paulo, p.100-103.

RATTER, J.A. 1987. Notes on the vegetation of the Parque Nacional do Araguaia (Brazil). Notes from the Royal Botanic Garden of Edinburg 44:311-342.

RATTER, J.A. \& DARGIE, C.D. 1992. An analysis of the floristic composition of 26 cerrado areas in Brazil. Edinburg Journal of Botany 49:235-250.

RATTER, J.A., POTT, A., POTT, V.J., CUNHA, C.N. \& HARIDASAN, M. 1988. Observations on woody vegetation types in the Pantanal and at Corumbá, Brazil. Notes from the Royal Botanic Garden of Edinburg 45:503-525.

RATTER, J.A., BRIDGEWATER, S., ATKINSON, R. \& RIBEIRO, J.F. 1996. Analysis of the floristic composition of the Brazilian cerrado vegetation II: comparison of the woody vegetation of 98 areas. Edinburg Journal of Botany 53:153-180.

RATTER, J.A., RIBEIRO, J.F. \& BRIDGEWATER, S. 1997. The Brazilian cerrado vegetation and threats to its biodiversity. Annals of Botany 80:223-230.

RATTER, J.A., BRIDGEWATER, S. \& RIBEIRO, J.F. 2003. Analysis of the floristic composition of the Brazilian cerrado vegetation III: comparison of the woody vegetation of 376 areas. Edinburgh Journal of Botany 60:57-109.

RAW, A. \& HAY, J. 1985. Fire and other factors affecting a population of Simarouba amara in "cerradão" near Brasília, Brazil. Revista Brasileira de Botânica 8:101-107.

RIBEIRO, J.F., SILVA, J.C.S. \& BATMANIAN, G.J. 1985. Fitossociologia de tipos físionômicos de cerrado em Planaltina, DF. Revista Brasileira de Botânica 8:131-142.

RICHARDS, A.J. 1986. Plant breeding systems. George Allen \& Unwin, London.

SARMIENTO, G. 1983. The savannas of tropical america. In Tropical savannas. Ecossystems of the world. 13 (F. Bouliére, ed.). Elsevier Science Publishers, New York, p.245-288. 
SILBERBAUER-GOTTSBERGER, I. \& EITEN, G. 1983. Fitossociologia de um hectare de cerrado. Brasil Florestal 54:55-70.

SILVA, L.O., COSTA, D.A., SANTO FILHO, K., FERREIRA, H.D. \& BRANDÃO, D. 2002. Levantamento florístico e fitossociológico em duas áreas de cerrado sensu stricto no Parque Estadual da Serra de Caldas Novas, Goiás. Acta Botanica Brasilica 16:43-53.
TOLEDO FILHO, D.V., LEITÃO FILHO, H.F. \& SHEPHERD, G.J. 1989. Estrutura fitossociológica da vegetação de cerrado em Mogi-Mirim (SP). Revista do Instituto Florestal 1:1-12.

WEISER, V.L. \& GODOY, S.A.P. 2001. Florística em um hectare de cerrado stricto sensu na ARIE - cerrado Pé-de-Gigante, Santa Rita do Passa Quatro, SP. Acta Botanica Brasilica 15:201-212. 